\title{
Editoriale
}

\section{Quale selvicoltura?}

\section{Roberto Del Favero}

\begin{abstract}
What kind of silviculture? This editorial stresses the need for a kind of silviculture that could address properly the profound changes in the wood market determined by the globalization of the economy and the most stringent environmental issues.
\end{abstract}

Keywords: Silviculture, Forest management, Wood market, Globalization.

Citation: Del Favero R, 2006. Quale selvicoltura? Forest@ 3 (1): 1-2. [online] URL: http://www.sisef.it/

Scorrendo la bibliografia forestale si può notare che, in corrispondenza d'importanti cambiamenti della società, compaiono spesso articoli che pongono la domanda: "quale selvicoltura?". In altre parole, in queste occasioni alcuni studiosi si sono posti il problema di mettere in discussione e riesaminare la validità delle idee e degli indirizzi selvicolturali. A titolo d'esempio, si possono citare i relativamente numerosi articoli sull'argomento comparsi fra gli anni settanta e ottanta del secolo scorso in occasione dell'affermarsi nella società di una maggiore sensibilità verso le tematiche ambientali.

E' il caso, oggi, di porsi un'analoga domanda? Credo che la riposta debba essere positiva. La globalizzazione dell'economia, che ha interessato in diversa misura tutti i settori produttivi, non ha risparmiato neppure quello dell'industria del legno, sempre più interessata da processi di delocalizzazione, verso i Paesi a basso costo, della materia prima e della manodopera. Questo ha portato a una drastica riduzione della domanda di prodotti legnosi nazionali, già di per se cronicamente limitata da varie cause (alto costo della manodopera, scarsa organizzazione del mercato, complessità delle procedure di autorizzazione, ecc.), facendo precipitare i prezzi di macchiatico e, di conseguenza, marginalizzando la produzione legnosa di molte proprietà forestali e quel poco di tessuto imprenditoriale ancora presente nel settore delle imprese boschive.

A fronte di questo quadro economicamente sconfortante si sono però aperte anche nuove prospettive inerenti la produzione legnosa. In primis, il crescente costo delle fonti energetiche tradizionali e la precarietà del loro approvvigionamento, nonché il miglioramento tecnologico e i seppur insufficienti incentivi a favore delle fonti energetiche alternative, hanno portato a un notevole incremento della domanda di legna come combustibile. L'interesse verso questo prodotto riguarda principalmente i proprietari privati, ma anche quelli pubblici, stimolati a trovare nuove fonti di entrata a seguito dei pesanti tagli dei trasferimenti dei fondi dallo Stato. Torna così a ridestarsi l'interesse anche per il governo a ceduo, spesso abbandonato da almeno un trentennio, sia per motivi economici sia perché considerato una coltura "di rapina".

Ma la globalizzazione dei mercati ha, fra i suoi paradossi, anche quello della valorizzazione delle specificità locali e della qualità. Nel settore della produzione legnosa questo si è manifestato con un incremento della domanda di legname di alta qualità per usi particolari. Si tratta evidentemente di piccoli quantitativi, capaci però di dar luogo a prodotti di nicchia altamente remunerativi e, perciò, fortemente attraenti, soprattutto per i proprietari privati, per questo incentivati a riprendere l'attività forestale, applicando soprattutto una sorta di "taglio saltuario commerciale", trattamento a suo tempo ritenuto anch'esso "di rapina", almeno se condotto con finalità esclusivamente "dimensionali-produttive".

Già queste sintetiche considerazioni vanno configurando nuove problematiche che giustificano pienamente la domanda: quale selvicoltura?

Come spesso accade nelle Società occidentali, anche per queste tematiche si possono delineare due opposte tendenze. La prima, che potremmo chiamare "protezionistica", vede favorevolmente la possibilità di ridurre ulteriormente il prelievo legnoso, configurando una selvicoltura, non tanto tesa a sostenere la produzione di materia prima, ma orientata 
a valorizzare le funzioni "ambientali" attribuite al bosco. Si tratta di una posizione condivisibile. Tuttavia, essa potrà risultare "sostenibile" solo individuando i soggetti che dovranno accollarsi i costi diretti e indiretti di tale selvicoltura. Oggi, nel contesto di un quadro economico difficile e complesso per il nostro Paese, non si può, infatti, ipotizzare che "la mano pubblica" possa da sola sostenere quest'onere e magari anche risarcire ai proprietari i mancati redditi.

La seconda tendenza, che potremmo chiamare "produttivistica", sarebbe invece favorevole a soddisfare incondizionatamente le "nuove" tendenze del mercato, ritenendo che, anche dal punto di vista socio-economico e ambientale, sia più opportuno conservare il tessuto imprenditoriale che gravita nell'area forestale piuttosto che rischiare un completo abbandono di ampi territori, incrementando i processi di urbanizzazione che oggi preoccupano non pochi Paesi. Tuttavia, il mero soddisfacimento delle richieste di mercato potrebbe comportare la ripresa di forme colturali ritenute ad alto impatto sulla funzionalità dei sistemi forestali.

Già questi brevi cenni delineano due concezioni opposte, che rischiano di entrare in contrapposizione, soprattutto se supportate dall'ideologia piuttosto che da concrete valutazioni oggettive. Tale contrapposizione, come purtroppo è spesso avvenuto nel mondo forestale nazionale, può portare a una sorta di "paralisi operativa", assolutamente da evitare se rapportata agli attuali ritmi evolutivi della Società.

Non è questa la sede per illustrare, anche solo sommariamente, le soluzioni a problematiche così complesse. Tuttavia, pare opportuno proporre una possibile soluzione che potrebbe configurasi nell' "innovazione", parola certamente abusata e sovente priva di contenuti. Molto spesso, infatti, lo stallo dovuto a contrapposte posizioni può essere su- perato, non tanto con confronti che si configurano spesso in scontri, ma portando "nuove" idee che, in genere, aumentano la qualità dell'approccio tecnico. E' quanto ha auspicato anche il Magnifico Rettore dell'Università di Firenze, Augusto Marinelli, quando, in occasione di un recente convegno svoltosi a Firenze, esortava i forestali a sviluppare nuove idee per affrontare un quadro economico-sociale in rapido cambiamento.

Le ipotesi di lavoro non mancano. Si può accennare così, a titolo d'esempio, alla possibilità di ridurre $\mathrm{i}$ costi di una selvicoltura con finalità soprattutto "ambientali" valorizzando il "vero" volontariato o comprendendo nel marketing territoriale anche le pratiche selvicolturali. Così pure non mancano le conoscenze per migliorare le tecniche colturali del governo a ceduo, riducendone l'impatto, o configurare linee colturali, già sperimentate in altri Paesi Europei, capaci di valorizzare la qualità del prodotto senza pregiudicare la funzionalità del sistema attraverso l'applicazione di interventi "eclettici", particolarmente adattati ad ogni singola realtà.

Già questi pochi esempi evidenziano la possibilità di attuare l'innovazione. Quello che è assolutamente da evitare è, invece, il timore di affrontare la sfida del cambiamento, radicandosi in vecchie impostazioni e/o sottovalutando i problemi che, invece, sono presenti poiché legati ai radicali cambiamenti che stanno interessando la nostra Società e, con essa, il mondo forestale.

\section{Author's Box}

Roberto Del Favero è ordinario di assestamento forestale e selvicoltura nell'Università di Padova; negli ultimi anni ha compiuto diversi studi sull'inquadramento della vegetazione forestale con finalità gestionali ed è stato autore di alcuni volumi sulle tipologie forestali, con particolare riferimento ai boschi della regione alpina;

E-mail: roberto.delfavero@unipd.it 